\title{
Interaction of thermal responsive NIPAM nanogels with model lipid monolayers at the air-water interface
}

\author{
Huihui Sun, Marina Resmini*, Ali Zarbakhsh* \\ Department of Chemistry and Biochemistry, SBCS, Queen Mary University of London, Mile \\ End Road, London E1 4NS, UK
}

E-mail: $\underline{\text { m.resmini@qmul.ac.uk and a.zarbakhsh@qmul.ac.uk }}$

Keywords: lipid monolayer, neutron reflectivity, nanogels, lipids-nanoparticles interactions

Understanding the interaction of nanoparticles (NP) with ceramide lipids is important in developing strategies to overcome the formidable obstacle that is skin. This paper presents studies of interactions between $\mathrm{N}$-isopropylacrylamide nanogels, crosslinked with 30\% N,N'methylenebisacrylamide, and model ceramide lipid monolayers at the air-water interface as a function of temperature. In the case of the mixed ceramide/cholesterol/behenic acid monolayer, the interaction of nanogels with the ceramide was strongly mediated by the fatty acids. This interaction between nanogels and monolayer components is dominated by hydrophobichydrophobic binding. The data show the important intermediary role of the fatty acid in facilitating transmembrane transport. For a pure ceramide lipid monolayer, the neutron reflectivity (NR), Brewster angle microscopy (BAM) and surface pressure results showed a lipid-nanogel complex formation and the subsequent depletion/solubilisation of the lipids from the interface when the area per molecule for the lipid was increased from 42 to $44 \AA^{2}$.

\section{Introduction}

The application of nanoparticles as delivery vehicles for small molecules and large biotherapeutics requires fundamental understanding of the nature of their interactions with biological membranes, characterized by their heterogeneous complex structures and varied permeation mechanisms. Among the different approaches, dermal drug delivery offers attractive 
advantages such as higher patient compliance and avoidance of first pass metabolism. However efficacy of the delivery route is dependent on permeation of the Stratum Corneum (SC) ${ }^{1}$, the natural skin barrier, and this remains a formidable challenge, requiring an understanding of the underlying mechanisms of interactions. Transport studies have shown that the lipid matrix of the SC, a multi-lamellar organized membrane, ${ }^{2}$ is the major diffusion-rate limiting pathway. ${ }^{3-5}$ The composition of the lipid matrix is very complex ${ }^{2}$; in addition to ceramide lipids and cholesterols there is a selection of other molecules such as fatty acids with chain lengths $\mathrm{C}_{14}$ up to $\mathrm{C}_{30}$. A number of different skin models have been reported in literature and, although there are some minor differences, it is commonly accepted that ceramide, cholesterol and free fatty acids with a molar ratio of 1:1:1 can offer a good model system ${ }^{6}$.

Among the different nanoparticles of interest, nanogels have attracted considerable attention because of their crosslinked yet flexible structures, with the ability to form stable colloidal solutions $^{7-10}$ and encapsulate both small and large molecules and tailor their release ${ }^{11-14}$. The choice of monomers and crosslinkers allows the tailoring of both chemical structure and physicochemical properties, leading, for example, to stimuli-responsive matrices hence having the ability to undergo rapid conformational changes ${ }^{15-19}$ altering their transmembrane transport. In particular, the hydrophobic interactions occurring at the lipid interface characteristic of skin is expected to accelerate their applications. ${ }^{20}$ Progress is being made to resolve the complex structure of biological membrane, for example Neutron Reflectivity (NR) studies of supported multi-layer lipid structures and their structural change as a result of inclusion of DNA have been reported $^{21}$. Other novel techniques such as combination of Molecular Dynamic simulations and Neutron Reflectivity for characterization of lipid bilayers have also been recently reported ${ }^{22}$. It has been shown that a spread lipid monolayer at the air-water interface is a suitable model for 
resolving the amphiphilic type interactions at the lipid interface. ${ }^{23}$ Therefore, the structural studies of nanogels and evaluation of their interactions with idealised lipid monolayers at the airwater interface, on the atomic length scale, can contribute to the understanding of the factors that facilitate permeation of nanoparticles through biological barriers such as the skin.

$\mathrm{N}$-isopropylacrylamide (NIPAM) is one of the most commonly used monomers for the preparation of thermoresponsive polymers. Linear pNIPAM has a lower critical solution temperature (LCST) of $32^{\circ} \mathrm{C}$, however the introduction of different combinations of comonomers and crosslinkers allows tailoring of the volume phase transition temperature (VPTT) of the polymers to suit different applications. ${ }^{24}$ The application of NIPAM microgels for dermal drug delivery has been investigated with interesting results; however there is still a limited understanding of the behavior of these nanoparticles at the interfaces and the study of the interactions with lipid layers. ${ }^{25-30}$

Previous work in our group demonstrated that ${ }^{31}$ NIPAM-based nanogels form collapsed monolayers at the air-water interfaces, and the thickness of the layer increases with the rigidity of the polymers as a result of higher crosslinker content.

In this paper we focus on interaction studies of NIPAM nanogels, crosslinked with $30 \%$ $N, N$ '-methylenebisacrylamide, with various monolayers consisting of either individual or mixed skin lipid species, i.e. a pure ceramide lipid monolayer, a behenic acid monolayer and a ceramide/cholesterol/behenic acid (molar ratio 1:1:1) mixed lipid monolayer. Behenic acid was chosen because of its availability in deuterated form. The $30 \%$ crosslinked NIPAM gel is a suitable candidate for drug loading, given the transition temperature of $38^{\circ} \mathrm{C}$, close to physiological temperature. The interactions of nanogels with the different layers were investigated at the air-water interface as a function of temperature using a combination of 
neutron reflectivity ${ }^{32-35}(\mathrm{NR})$, Brewster Angle Microscopy (BAM) and surface pressure measurements.

\section{Experimental Section}

\section{Chemicals and materials}

$N$-isopropylacrylamide (NIPAM, 97\%), $N, N^{\prime}$-methylenebisacrylamide (MBA, 99\%), anhydrous dimethyl sulfoxide (DMSO, 99.8\%), cholesterol (99\%), protonated behenic acid (99\%) and $\mathrm{D}_{2} \mathrm{O}$ were purchased from Sigma Aldrich and used as provided. 2,2'-Azobis(isobutyro)nitrile (AIBN, 98\%, Acros) was recrystallized from methanol. Both protonated C16 ceramide lipid, Npalmitoyl-D-erythro-sphingosine, and deuterated C16 ceramide lipid, N-palmitoyl-d31-Derythro-sphingosine with one stearoyl chains deuterated $\left(\mathrm{d}_{31}\right)$, were obtained from Avanti Polar Lipids, Inc. Deuterated behenic acid (behenic- $\mathrm{d}_{43}$ acid, $\mathrm{CD}_{3}\left(\mathrm{CD}_{2}\right)_{20} \mathrm{COOH}$ ) was purchased from $\mathrm{C} / \mathrm{D} / \mathrm{N}$ Isotopes, distributed by $\mathrm{QMX}$ Laboratories Ltd. The aqueous phases used had a $\mathrm{pH}$ around 7.0. The ultrapure deionized water was prepared by the Purelab option water purification unit (Elgastat). Dialysis membranes (22 mm diameter, MWCO $3500 \mathrm{Da}$ ) were brought from Medicell International Ltd. The synthesis and characterization of nanogels, the measurements of surface pressure molecular area $(\Pi-A)$ isotherms of ceramide lipid and behenic acid as well as BAM measurements are described in details in supporting information.

\section{Neutron reflectivity}

Neutron reflectivity (NR) measurements were carried out at the ISIS Spallation Neutron Source, Rutherford Appleton Laboratory, Didcot, UK using the SURF reflectometer ${ }^{36}$ and the Nima trough equipped with a Wilhelmy plate, providing a surface pressure profile. SURF is a time-offlight reflectometer with a $50 \mathrm{~Hz}$ chopper, selecting a wavelength range between 0.5 and $7.0 \AA$. The incoming beam comes at a fixed angle of incident $\theta=1.5^{\circ}$. The reflectivity $R\left(Q_{z}\right)$ was 
measured using the time-of-flight technique as a function of momentum transfer normal to the interface $Q_{z}=(4 \pi \sin \theta) / \lambda$, where $\theta$ is the grazing angle of incidence neutrons with wavelength $\lambda$ to provide the most suitable $Q$-range possible and the highest sensitivity to interfacial structure. The sample was under illuminated with resolution $\mathrm{d} Q / Q \sim 3 \%$. The average area of the trough was varied in the range of $130-490 \mathrm{~cm}^{2}$ by two computer-controlled moveable barriers. The neutron beams used to measure the reflectivity profiles had a constant footprint of $30 \mathrm{~cm}^{2}$, under illuminating the surface of the trough consequently, the structural details deduced from the NR data were averaged over a large macroscopic length scale.

The adsorption of $30 \mathrm{MBA}$ nanogels at the air-water interface was first studied as a function of temperature. The trough was filled with a nanogel dispersion with a concentration of $0.005 \mathrm{mg}$ $\mathrm{mL}^{-1}$ and a series of reflectivity profiles were measured as a function of temperature for a constant trough area. The system was then cooled down to $25^{\circ} \mathrm{C}$ in order to study the reversibility of the conformational changes of nanogels as a result of variations in temperature. $\mathrm{D}_{2} \mathrm{O}$ was used as the bulk aqueous phase to highlight the adsorption of hydrogenous nanogels at the interface.

For monolayer studies, d31-C16 ceramide lipid monolayer, mixed lipid monolayers of d31-C16 ceramide lipid/h-cholesterol/h- (or d43-) behenic acid (molar ratio 1:1:1) and d43-behenic acid monolayer were spread at the air-water interface. After the characterization of monolayers, a known amount of nanogels was then injected underneath the monolayers (final nanogel bulk concentration $0.005 \mathrm{mg} \mathrm{mL}^{-1}$ ) to investigate the interactions of the nanogels with various monolayers. Null reflecting water, NRW $\left(8.1 \%\right.$ vol of $\mathrm{D}_{2} \mathrm{O}$ in $\mathrm{H}_{2} \mathrm{O}$ giving a scattering length density equal to zero) was used as the aqueous sub-phase for all the measurements which provides an appropriate contrast, sensitive to the interaction of protonated nanogels with various 
deuterated lipid monolayers. The reflectivity profiles of all the monolayers before and after the addition of nanogels were measured as a function of temperature. All the NR measurements were performed by allowing the system to reach the meso-equilibrium state by monitoring the surface pressure simultaneously. The meso-equilibrium state is used to infer the state prior to full equilibrium of surface pressure. This state is taken as the stability of the surface pressure during measurement to within $\pm 3 \mathrm{mN} \mathrm{m}^{-1}$ prior to the neutron data collection. The molecular structures and scattering length density $N b$ of the lipids used for these experiments are shown in Fig. S1 and Table S1.

NR technique gives information on the neutron refractive index profile normal to an interface relating to concentration/composition at the interface on an Angström length scale. The neutron refractive index for soft matters (absence of any strong absorber such as cadmium) can be expressed as follow:

$$
n=1-\frac{N b}{2 \pi} \lambda^{2}
$$

where $N$ is the atomic number density, $b$ is the bound coherent scattering length. The $N b$ is defined as scattering length density and varies proportionally with the volume fraction of the components $^{37}$ :

$$
N b=\sum_{i} \varphi_{i} N b_{i}
$$

where $\varphi_{i}$ and $N b_{i}$ are the volume fraction and scattering length density of component $i$, respectively. The sum of the volume fractions for all the species is $1\left(\sum_{i} \Phi_{i}=1\right)$.

The NR data were analysed by using the optical matrix method. ${ }^{38}$ The structure of the interface is described as a series of discrete layers, each defined in terms of $N b$, thickness $(d)$, and interfacial roughness $(\sigma)$ with the next layer. 


\section{Results and discussion}

Previous work demonstrated a correlation between the degree of crosslinker present in the NIPAM-based nanogels and physico-chemical characteristics, including the VPTT, in addition to a thicker layer formed at the air/water interface. On that basis NIPAM nanogels were prepared with $30 \%$ MBA as cross-linker, following our previously reported methodology, ${ }^{31}$ and were obtained with $73 \%$ yield. In the first instance the nanogels' VPTT was evaluated by UV-Vis spectroscopy, which confirmed a value of $38^{\circ} \mathrm{C}$ close to physiological body temperature, making these materials suitable for dermal drug delivery applications. Particle size was characterized (Table 1) using DLS, in the bulk solution, and confirmed by TEM. The average particle size for 30MBA was found to be $\sim 12 \mathrm{~nm}$ (with zeta potential of $\sim-0.15 \mathrm{mV}$ ) at room temperature, and remained constant up to VPTT. The prepared nanogels were approximately spherical in shape as shown by the TEM image (Fig. S2).

Table 1. Characterization of nanogels $30 \mathrm{MBA}$ with calculated value of nanogel scattering length density, $N b$.

\begin{tabular}{|c|c|c|c|c|c|c|c|}
\hline Sample & $\begin{array}{l}\% \mathrm{~mol} \\
\mathrm{MBA}\end{array}$ & $Y, \%$ & $d_{h}, \mathrm{~nm}$ & $P d I$ & $\rho, \mathrm{g} \mathrm{cm}^{-3}$ & $\begin{array}{c}N b \times 10^{-6} \\
\AA^{-2}\end{array}$ & $V P T T,{ }^{\circ} \mathrm{C}$ \\
\hline $30 \mathrm{MBA}$ & 30 & 73 & $11.9 \pm 0.4$ & 0.37 & 1.7 & 1.76 & 38 \\
\hline
\end{tabular}

\section{Nanogels at the air-water interface}

The main focus here is to resolve first the structure of $30 \mathrm{MBA}$ nanogels and spread ceramide monolayer alone at the air-water interface perpendicular to the interface, followed by structural changes of the lipid monolayer in the presence of nanogels at the interface. The adsorption of 
$30 \mathrm{MBA}$ at the air-water interface was first investigated by NR, surface pressure and BAM prior to studies of its interactions with lipid monolayers. The normalised reflectivity profiles as a function of temperature from 19 to $44^{\circ} \mathrm{C}$ are shown in Fig. 1a. The reflectivity was found to decrease as a function of temperature. Fig. 1b shows a clear reversibility of nanogels conformation on the heating and cooling cycles, which is also confirmed by the surface pressure data. It was found that a minimum of a two-layer model is required to obtain consistent fits to the datasets for all temperatures. The fits to the reflectivity profiles are represented by the solid lines in Fig. 1a inset and the corresponding parameters are given in Table 2.

The fitting model indicates that the adsorbed nanogels at the air-water interface consist of two distinct layers, a first thin collapsed layer in contact with air and a second much thicker solvated layer immersed in the bulk aqueous phase. The second layer at the interface with the aqueous bulk water is roughened. It reflects the dynamic accumulation of nanogels at the layer/bulk interface. It is primarily because of the assembly of new materials arriving from the bulk. Previous electron microscopy studies of NIPAM microgels at the hydrophobic-hydrophilic (oilwater) interface have shown that a large proportion of the particles tend to reside on the aqueous bulk side of the interface while a small amount protruded into the oil (hydrophobic) phase..$^{39-42}$ Reasonable assumptions were required in order to estimate the volume fraction of nanogels $\left(\varphi_{\text {nanogel }}\right)$ in each layer; the first layer consists of nanogels and air while the second layer is made up of nanogels and water. The calculated results (according to Equation 2) are shown in Table 2. The enrichment of the polymer composition of both layers and the steady increase in the second layer thickness from $36 \AA$ to $46 \AA$ as temperature rises are indicative of an increasing amount of nanogels being adsorbed at the interface and hence a greater surface activity of nanogels at higher temperature as expected. This is further supported by the BAM (Fig. S3) and surface 
pressure data (Fig. S4). Although the BAM image intensities are not quantitative, they give an indication of how the monolayer density is changing. It can be clearly seen that the images are brighter, revealing an increasing amount of nanogels adsorbed at the air-water interface as the temperature increases. These results are in agreement with literature data reported for both linear polymer and microgels. ${ }^{43-45}$ The increased surface activity of nanogels is driven by the loss of the hydration shell (i.e. increased degree of hydrophobicity) as temperature increases.
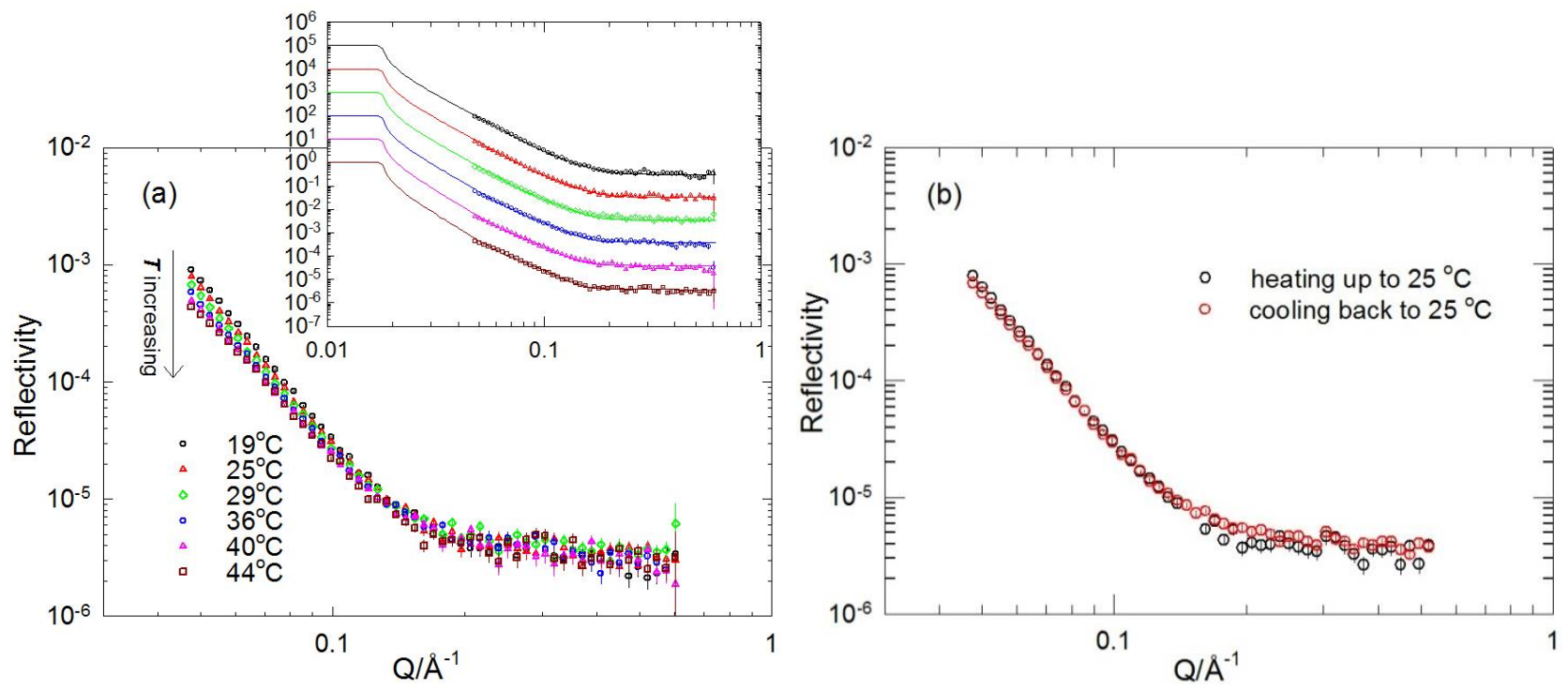

Fig. 1 a) Reflectivity spectra $R(Q)$ of nanogels $30 \mathrm{MBA}$ at the air- $\mathrm{D}_{2} \mathrm{O}$ interface for a series of temperatures. The inset shows the shifted profiles for the purpose of clarity. The solid lines are fits to the data using two-layer model. b) Reflectivity profiles measured at the same temperature during heating up and cooling process. Bulk concentration is equal to $0.005 \mathrm{mg} \mathrm{ml}^{-1}$.

Table 2 Parameters used for two-layer model fits to $30 \mathrm{MBA}$ nanogels at the air- $\mathrm{D}_{2} \mathrm{O}$ interface. The calculated fits are shown by solid lines in Fig. 2a inset.

\begin{tabular}{cccccc}
\hline $\begin{array}{c}\text { Temp, } \\
\pm 1{ }^{\circ} \mathrm{C}\end{array}$ & $\begin{array}{c}d, \AA \\
\pm 1\end{array}$ & $\begin{array}{c}N b_{\text {fitted }} \times 10^{-6} \AA^{-2} \\
\pm 0.1\end{array}$ & $\begin{array}{c}\sigma, \AA \\
\pm 1\end{array}$ & $\begin{array}{c}\text { Air-film } \\
\text { roughness, } \AA \\
\pm 1\end{array}$ & $\varphi_{\text {nanogel }} \%$ \\
\hline 19 & 17 & 1.0 & 4 & 5 & 58
\end{tabular}




\begin{tabular}{|c|c|c|c|c|c|}
\hline & 36 & 5.8 & 5 & & 12 \\
\hline 25 & $\begin{array}{l}17 \\
38\end{array}$ & $\begin{array}{l}1.1 \\
5.7\end{array}$ & $\begin{array}{l}4 \\
8\end{array}$ & 5 & $\begin{array}{l}64 \\
15\end{array}$ \\
\hline 29 & $\begin{array}{l}17 \\
40\end{array}$ & $\begin{array}{l}1.2 \\
5.5\end{array}$ & $\begin{array}{c}4 \\
10\end{array}$ & 5 & $\begin{array}{l}69 \\
19\end{array}$ \\
\hline 36 & $\begin{array}{l}17 \\
43\end{array}$ & $\begin{array}{l}1.3 \\
5.3\end{array}$ & $\begin{array}{c}4 \\
12\end{array}$ & 5 & $\begin{array}{l}77 \\
22\end{array}$ \\
\hline 40 & $\begin{array}{l}17 \\
46\end{array}$ & $\begin{array}{l}1.4 \\
5.2\end{array}$ & $\begin{array}{c}4 \\
15\end{array}$ & 5 & $\begin{array}{l}80 \\
25\end{array}$ \\
\hline 44 & $\begin{array}{l}17 \\
46\end{array}$ & $\begin{array}{l}1.5 \\
5.1\end{array}$ & $\begin{array}{c}4 \\
15\end{array}$ & 5 & $\begin{array}{l}84 \\
27\end{array}$ \\
\hline
\end{tabular}

\section{Interaction of nanogels with lipid monolayers}

In order to better understand the interaction mechanism of NIPAM based nanogels with lipid model monolayers we have used NR and BAM to gain more information on a molecular level. In these experiments, three different model monolayers, i.e. pure ceramide lipid monolayer, ceramide/cholesterol/behenic acid (molar ratio 1:1:1) mixed lipid monolayer and behenic acid monolayer, were used.

\section{Pure ceramide lipid monolayer}

The stability of a spread bare d-ceramide (d-cer) lipid monolayer as a function of temperature was first investigated by $\mathrm{NR}$ and BAM using a Langmuir trough at $31.0 \mathrm{mN} \mathrm{m}^{-1}$ (molecular area of $42 \AA^{2}$ ). This surface pressure arouses interests as it is considered to be the lateral pressure in erythrocyte membranes. ${ }^{46}$ Although NR is a sensitive technique for determining the composition and the density profiles normal to the interface, it does not provide lateral information such as phase changes and in-plane structures. Hence BAM was used as a complementary technique to 
further characterize any possible phase change in the spread lipid monolayer. Both NR and BAM data (Fig. S5 and S6) suggest that the pure ceramide lipid monolayer was stable and no changes in the monolayer morphology were observed over the temperature range of $20-44{ }^{\circ} \mathrm{C}$, which is in good agreement with the literature. ${ }^{48}$ BAM data showed in-plane structure from the ceramide monolayer because of the asymmetric nature of the tails resulting in domain formations. The contribution to the background is affecting the data across the Q-range; this includes both incoherent, in-plane off-specular contributions and inherent instrumental background. However it is significantly more notable at larger $\mathrm{Q}$ because the intensities are much lower. The background is difficult to model and is fitted as a flat line across all the Q. However it is also important to note that neutron (specular) reflectivity is sensitive to structural details perpendicular to a given interface only. The NR data at $20{ }^{\circ} \mathrm{C}$ is fitted to a single layer of $24 \AA$ (Fig. S5). The fitted layer thickness is comparable to the length of a C16 ceramide molecule oriented away from the surface. The volume fraction of d-ceramide lipid $\left(\varphi_{d-c e r}\right)$ was calculated to be $99 \%$ (Equation 2). This high value suggests a compact lipid monolayer at the interface. The results indicate that a well packed and ordered ceramide lipid monolayer with the lipid molecules oriented normal to the air-water interface was formed ${ }^{49}$ at $31.0 \mathrm{mN} \mathrm{m}^{-1}$ as expected.

\section{Interaction of nanogels with pure ceramide lipid monolayer}

The interaction of nanogels with pure d-cer lipid monolayer was then investigated as a function of temperature from 25 to $42{ }^{\circ} \mathrm{C}$. Nanogels $30 \mathrm{MBA}$ were injected slowly underneath the monolayer from outside the barriers at $31.0 \mathrm{mN} \mathrm{m}^{-1}$ and $25{ }^{\circ} \mathrm{C}$. The NR data are shown in Fig. 2a. There is no change in reflectivity profiles detected at this pressure over the investigated temperature range. This is further confirmed by the BAM measurement (Fig. S7). The surface pressure data however, shows a change (Fig. 2b). It can be seen that after the addition of 
nanogels at $25{ }^{\circ} \mathrm{C}$, the surface pressure increased from 31.0 to $32.0 \mathrm{mN} \mathrm{m}^{-1}$ and then this value gradually reached $42.5 \mathrm{mN} \mathrm{m}^{-1}$ as the temperature was raised stepwise to $42{ }^{\circ} \mathrm{C}$. This change in surface pressure was found to be reversible on cooling to $23{ }^{\circ} \mathrm{C}\left(\sim 31.2 \mathrm{mN} \mathrm{m}^{-1}\right)$.

The surface pressure changes on one hand suggest increasing accumulation of nanogels underneath the lipid monolayer (not visible to BAM and NR), as a function of temperature. On the other hand, the reversibility of the surface pressure on the cooling cycle indicates clearly no strong interaction between the mainly hydrophobic nanogels and the highly compact lipid monolayer (head groups).
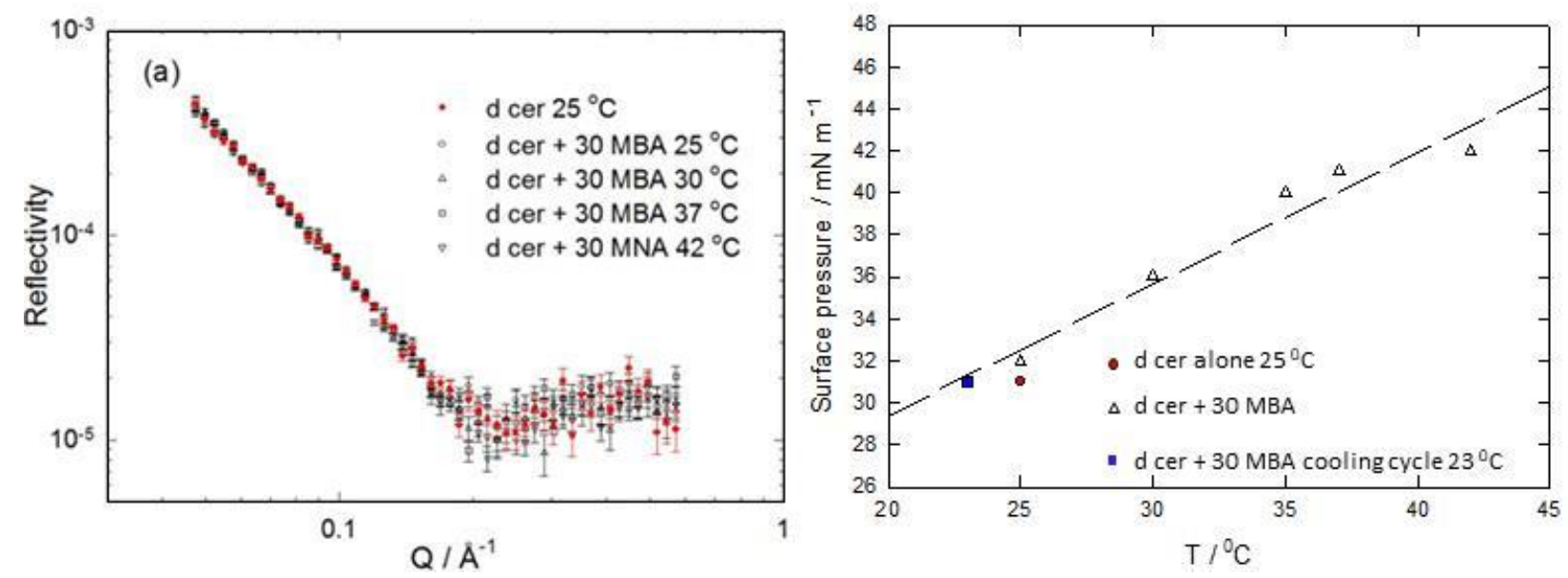

Fig. 2 a) Reflectivity spectra $R(Q)$ from d-cer monolayer (spread at $31.0 \mathrm{mN} \mathrm{m}^{-1}$ ) at the air-NRW interface for a series of temperatures in the absence and the presence of nanogels $30 \mathrm{MBA} . \mathrm{b}$ ) Surface pressure $\Pi$ of lipid monolayer without and with the presence of $30 \mathrm{MBA}$ at different temperatures. The system was cooled back to $23^{\circ} \mathrm{C}$ after heating.

As mentioned above, one promising application of these thermo-responsive nanogels is as dermal drug delivery systems. The interaction of nanogels $30 \mathrm{MBA}$ with a pure ceramide lipid monolayer was studied at a surface pressure of $20.0 \mathrm{mN} \mathrm{m}^{-1}$ (molecular area of $44 \AA^{2}$ ). This lower surface pressure (higher molecular area) has been suggested to result in the coexistence of 
a highly crystalline phase with a loosely packed amorphous phase in the monolayer. ${ }^{49}$ Fig. 3 shows the reflectivity profiles of this monolayer without and with the presence of $30 \mathrm{MBA}$ at this pressure as a function of temperature. The reflectivity decreases after the addition of $30 \mathrm{MBA}$, suggesting an interaction of these nanogels with the lipid monolayer and this interaction being temperature dependent. It was found that the reflectivity profile of the bare ceramide lipid monolayer can be represented by a one-layer model, while a two-layer model is required to obtain a reasonable representation for the datasets obtained after the addition of $30 \mathrm{MBA}$. The fits are represented by the solid lines in Fig. 3a. The corresponding $N b$ profiles are shown in Fig. $3 \mathrm{~b}$ and the fitted parameters are given in Table 3. The decrease in $N b$ of the first layer from $3.4 \times 10^{-6} \AA^{-2}$ to $3.1 \times 10^{-6} \AA^{-2}$ after the addition of nanogels 30 MBA can be explained either (i) by the loss of deuterated ceramide lipids, as shown schematically in Fig. 3d or (ii) by the penetration of hydrogenous nanogels into the monolayer region as illustrated in Fig. 3e. The simulation for the latter model is represented by the dashed line in Fig. 3a inset. This clearly indicates that model (ii) is not adequate in representing the data and some degree of solubilization of deuterated lipids by nanogels must have taken place. As the temperature increases, the $\mathrm{Nb}$ of the first layer gradually decreases from $3.1 \times 10^{-6} \AA^{-2}$ to $2.0 \times 10^{-6} \AA^{-2}$, indicating an increasing amount of deuterated lipids were being depleted from the interface. The volume fraction of $\mathrm{d}$ ceramide lipid $\left(\varphi_{d-c e r}\right)$ in this layer was calculated (Equation 2) and is shown in Table 3. It can be seen that $42 \%$ of the d-ceramide lipid was depleted by nanogels at the highest temperature of 43 ${ }^{\circ} \mathrm{C}$. The presence of the second layer and the increase in its $\mathrm{Nb}$ value with temperature indicate the complex formation of protonated nanogels and deuterated lipids (Fig. 3d). This complex formation was found to be non-reversible with temperature (red symbols in Fig. 3a), suggesting a 
strong hydrophobic-hydrophobic interaction between nanogels and ceramide lipids (now in an amorphous phase).
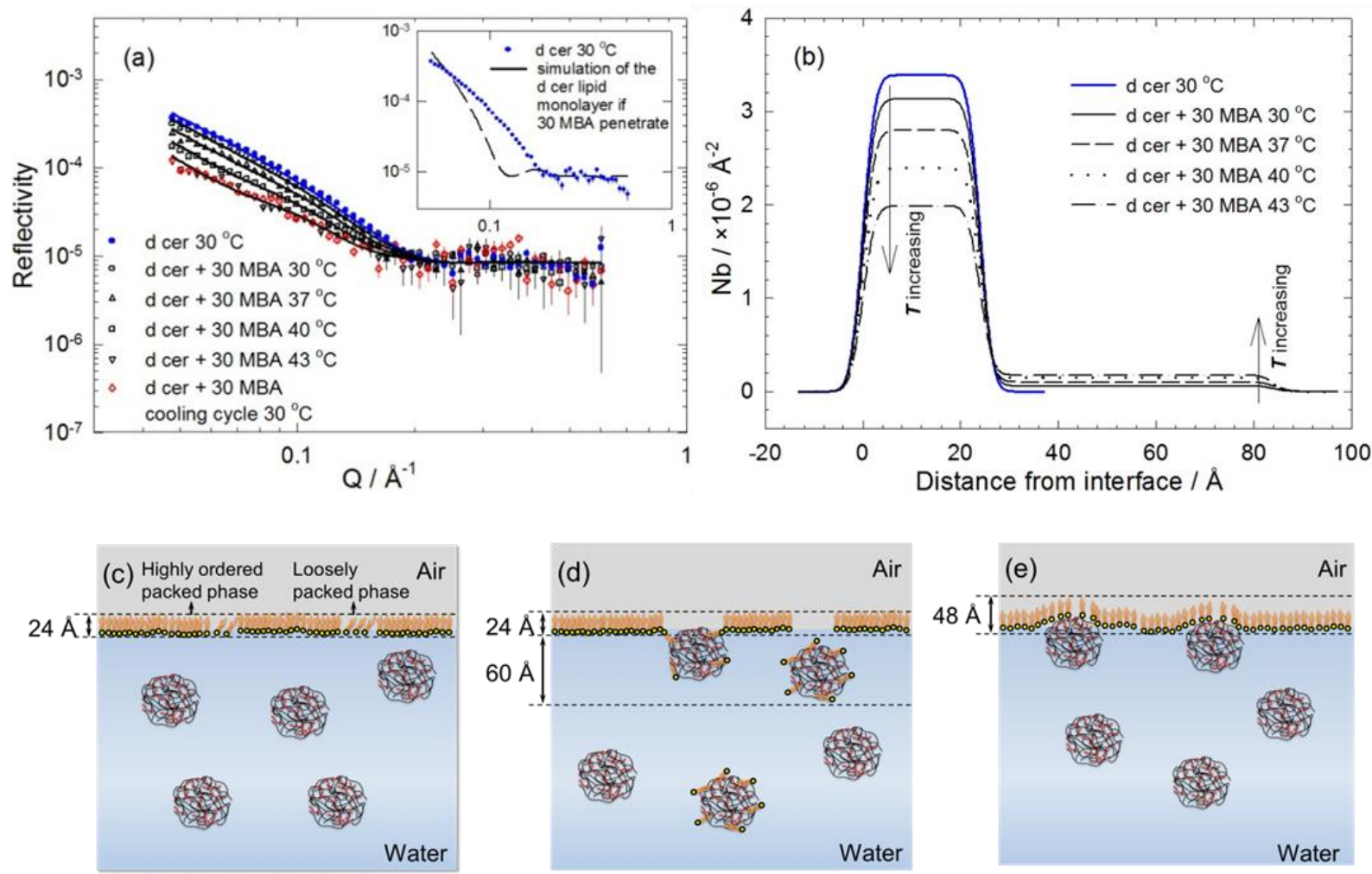

Fig. 3 a) Reflectivity spectra $R(Q)$ from the d-cer monolayer (spread at $20.0 \mathrm{mN} \mathrm{m}^{-1}$ ) at the airNRW interface for a series of temperatures without and with the presence of $30 \mathrm{MBA}$. The solid lines are fits to the data using one-layer model (blue) for bare lipid monolayer and two-layer model (black) after the addition of $30 \mathrm{MBA}$. The inset shows the simulation (dashed line) of the monolayer if the nanogels penetrate the lipid monolayer without depleting the lipids; b) Scattering length density $(\mathrm{Nb})$ profiles for calculated reflectivity profiles shown in a); Schematic diagrams show c) the packing of the bare ceramide lipid monolayer at the air-water interface at molecule area of $44 \AA^{2}\left(20.0 \mathrm{mN} \mathrm{m}^{-1}\right)$; d) the depletion of d-cer lipids by nanogels $30 \mathrm{MBA}$ to form water dispersible complexes which are adsorbed at the interface, corresponding to the fitted 
solid lines in a); e) the penetration of $30 \mathrm{MBA}$ through the lipid monolayer without depleting the lipids, corresponding to the simulation dashed line shown in a) inset.

Table 3. Parameters for fits in Fig. 3a

\begin{tabular}{lcccccc}
\hline Sample & $\begin{array}{c}\text { Temp, } \\
\pm 1^{\circ} \mathrm{C}\end{array}$ & $\begin{array}{c}d, \AA \\
\pm 1\end{array}$ & $\begin{array}{c}N b_{\text {fitted }} \times 10^{-6} \AA^{-2} \\
\pm 0.1\end{array}$ & $\begin{array}{c}\sigma, \AA \\
\pm 1\end{array}$ & $\begin{array}{c}\text { Air-film } \\
\text { roughness, } \\
\AA \\
\pm 1\end{array}$ & $\varphi_{\text {d-cer }, \%}$ \\
\hline d-cer monolayer & 30 & 24 & 3.4 & 2 & 2 & 99 \\
d-cer monolayer + 30 MBA & 30 & 24 & 3.1 & 2 & 2 & 91 \\
d-cer monolayer + 30 MBA & 37 & 24 & 2.8 & 2 & 2 & 81 \\
& & 60 & 0.1 & 2 & & $\mathrm{nc}$ \\
d-cer monolayer + 30 MBA & 40 & 24 & 2.4 & 2 & 2 & 69 \\
& & 60 & 0.1 & 2 & & $\mathrm{nc}$ \\
d-cer monolayer + 30 MBA & 43 & 24 & 2.0 & 2 & 2 & 58 \\
& & 60 & 0.2 & 2 & & $\mathrm{nc}$ \\
\hline
\end{tabular}

$d$ - layer thickness, $N b_{\text {fitted }}$ - fitted scattering length density, $\sigma$ - roughness, $\varphi_{d-c e r}$ - volume fraction of d-ceramide; nc - not calculable

\section{Mixed lipid monolayer of ceramide/cholesterol/behenic acid}

In order to mimic a more realistic membrane, the mixture of ceramide/cholesterol/behenic acid (molar ratio 1:1:1) was used to form a monolayer. The thermo-stability of this mixed lipid monolayer at the surface pressure of $31.0 \mathrm{mN} \mathrm{m}^{-1}$ was examined over the temperature range of $25-43{ }^{\circ} \mathrm{C}$ by both NR and BAM. Two different contrasts were used for NR measurements, i.e. d-ceramide/h-cholesterol/h-behenic acid (d-cer/h-chol/h-BA) and d-ceramide/h-cholesterol/dbehenic acid (d-cer/h-chol/d-BA). The normalised NR profiles as a function of temperature for 
both contrasts are shown in Fig. S8. Both sets of contrasts show that there is no change in the conformation of the mixed lipid monolayer as a function of temperature $\left(25\right.$ to $\left.43{ }^{\circ} \mathrm{C}\right)$.

Both contrasts can be reasonably represented by a one-layer model and the fits are shown by solid lines together with the fitted parameters, in Fig. S8a-b. The fitted layer thickness of the mixed lipid monolayer $(28 \AA)$ is slightly thicker than the one of the pure ceramide lipid monolayer (24 $\AA$ ), as a result of behenic acid, which has a longer hydrocarbon chain length. The fitted $N b$ of the mixed lipid monolayer for $\mathrm{d}$-cer $/ \mathrm{h}$-chol/h-BA and d-cer $/ \mathrm{h}-\mathrm{chol} / \mathrm{d}-\mathrm{BA}$ is $1.3 \times 10^{-6}$ $\AA^{-2}$ and $2.1 \times 10^{-6} \AA^{-2}$, respectively. Assuming that the three components in the spread mixed monolayer are miscible and form an homogenous phase, the sum of $\varphi_{\text {air }}$ and $\varphi_{\text {water }}$ (i.e. $\left.\varphi_{a i r}+\varphi_{\text {water }}\right)$ could be calculated according to Equation 2, being $12 \%$ for contrast d-cer $/ \mathrm{h}$-chol $/ \mathrm{h}$ BA and $36 \%$ for another contrast $\mathrm{d}-\mathrm{cer} / \mathrm{h}-\mathrm{chol} / \mathrm{d}-\mathrm{BA}$. The calculated $\varphi_{\text {air }}+\varphi_{\text {water }}$ for the two contrasts should be equal to each other; however, there is a difference as large as $24 \%$, suggesting that the above assumption is not reasonable. As these three components are not entirely miscible at a molar ratio of 1:1:1, and phase separation occurs, the coexistence of different phases can be hypothesized.

The above conclusion is strongly supported by BAM images (Fig. 4), which show the coexistence of three phases in the mixed lipid monolayer, namely the thin, large domains (grey) of irregular shape and the thicker, small domains (brighter) embedded in a thin, flat monolayer (darker). According to the brightness of the above three different phases, they are assigned as rich in ceramide, behenic acid, and cholesterol, respectively. As temperature increases from 25 to $37^{\circ} \mathrm{C}$, clear phase changes can be observed in the BAM images where there are more phases rich in behenic acid (the brightest domains). This could be attributed to the large difference in hydrocarbon chain lengths between behenic acid and the other two components. Since the mixed 
lipid monolayer is not stable with temperature, by showing an obvious phase separation, the interaction of nanogels $30 \mathrm{MBA}$ with the mixed lipid monolayer $\left(\Pi=31.0 \mathrm{mN} \mathrm{m}^{-1}\right)$ was studied at $37^{\circ} \mathrm{C}$ which is close to the physiological body temperature.

(a) Blank air-water interface

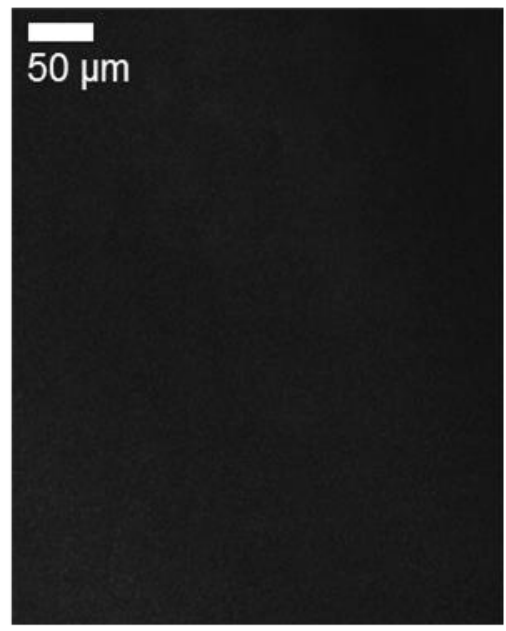

(c) Mixed lipid monolayer

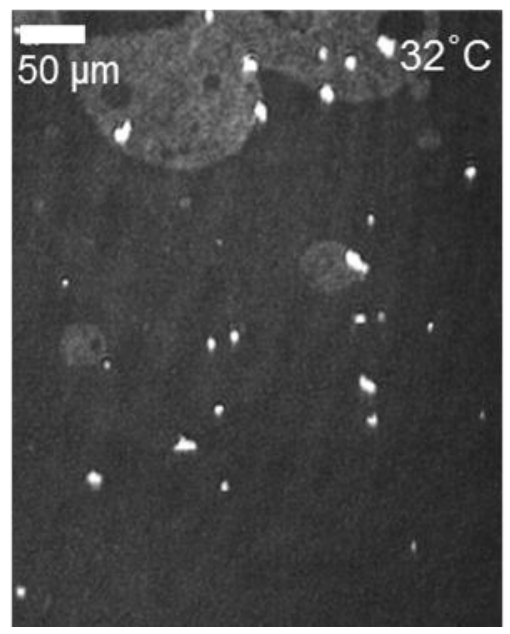

(b) Mixed lipid monolayer

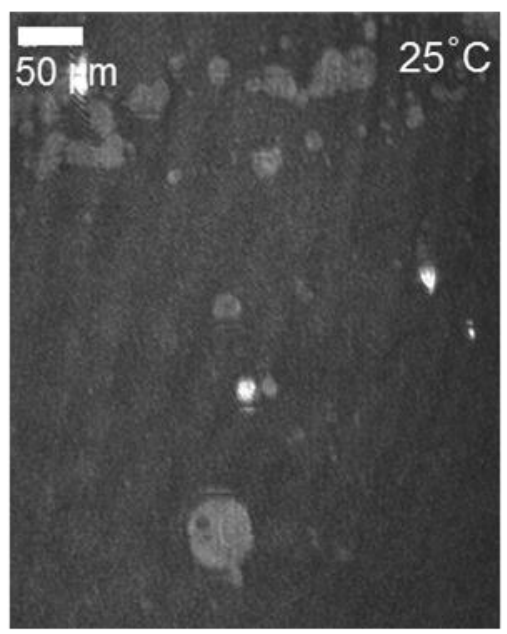

(d) Mixed lipid monolayer

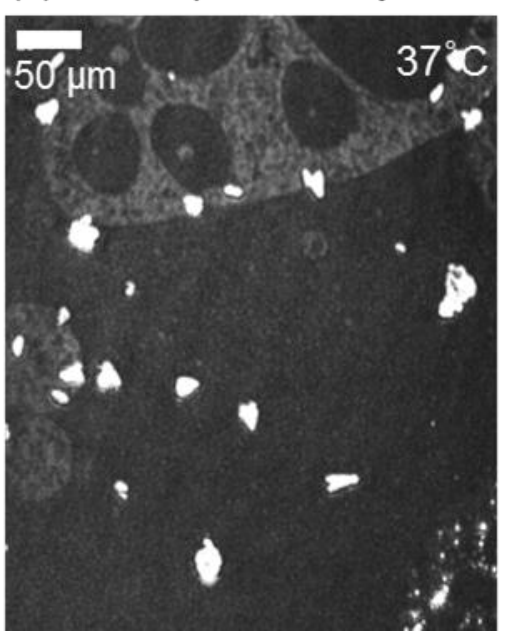

Fig. 4 BAM images of the air-water interface in presence of mixed lipid monolayer $(\Pi=31.0 \mathrm{mN}$ $\mathrm{m}^{-1}$ ) composed of ceramide/cholesterol/behenic acid with molar ratio of $1: 1: 1$ at different temperature. a) blank air-water interface as a reference, b) $25^{\circ} \mathrm{C}$, c) $32{ }^{\circ} \mathrm{C}$ and d) $37^{\circ} \mathrm{C}$.

Interaction of nanogels with mixed lipid monolayer of ceramide/cholesterol/behenic acid 
The normalised NR profiles of the mixed lipid monolayer (spread at $31.0 \mathrm{mN} \mathrm{m}^{-1}$ ) with two different contrasts before and after the addition of $30 \mathrm{MBA}$ are shown in Fig. 5. All the profiles can be described with a one-layer model and the fit is represented by solid lines, in Fig. 5a-b for the two contrasts respectively. The resulting parameters are presented in Fig. 5a and Table 4. For the $\mathrm{d}-\mathrm{cer} / \mathrm{h}$-chol/h-BA, no change in the reflectivity profile can be observed after the addition of 30 MBA. On the contrary, there is an obvious decrease in the reflectivity of the $\mathrm{d}$-cer $/ \mathrm{h}-\mathrm{chol} / \mathrm{d}$ BA, suggesting a depletion of materials from the interface. By comparing the two contrasts it can be deduced that the decrease in reflectivity in Fig. $5 \mathrm{~b}$ is mainly caused by the depletion of deuterated behenic acid. In the first contrast, the behenic acid is protonated with $N b$ equal to $0.1 \times 10^{-6} \AA^{-2}$, which is close to the NRW. As a result, any loss of behenic acid is not detectable by the neutrons. In the second contrast, the h-behenic acid is replaced by d-behenic acid ( $\mathrm{Nb}=$ $5.7 \times 10^{-6} \AA^{-2}$ ); therefore, the depletion of it by the nanogels becomes visible to neutrons. The decrease in volume fraction of d-behenic acid $\left(\varphi_{\text {decreased }}\right)$ caused by the depletion is estimated to be $5 \%$ according to Equation 3:

$$
\varphi_{\text {decreased }}=\frac{N b_{\text {layer }}-N b_{\text {layer }}^{\prime}}{N b_{d-B A}}
$$

where $N b_{\text {layer }}$ and $N b_{\text {layer }}^{\prime}$ is the scattering length density of mixed lipid monolayer before and after the addition of $30 \mathrm{MBA}$, respectively, and $N b_{d-B A}$ is the scattering length density of dbehenic acid. The behenic acid is depleted from the interface in a similar way to that of the ceramide lipid as a result of hydrophobic-hydrophobic interaction with nanogels.

At $31.0 \mathrm{mN} \mathrm{m}^{-1}\left(37^{\circ} \mathrm{C}\right), 30 \mathrm{MBA}$ nanogels showed no interaction with the pure ceramide lipid monolayer (Fig. 2) whereas there is interaction now with a mixed lipid monolayer. This is believed to be the result of the increased fluidity of the mixed ceramide lipid layer (i.e. weaker inter-molecule binding) after the introduction of cholesterol and behenic acid. The finding is in 
good agreement with the literature ${ }^{50}$ which reported that the permeability of a lamellar membrane was regulated by the degree of its fluidity.
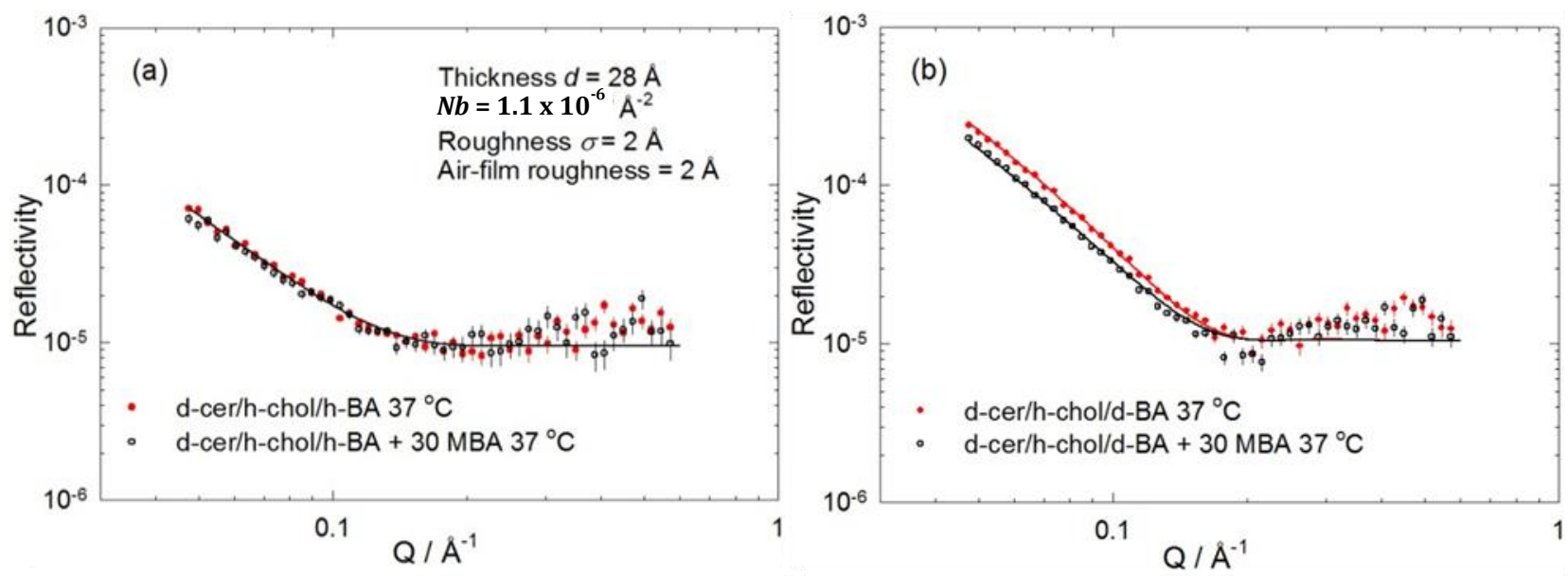

Fig. 5 Reflectivity spectra $R(Q)$ from the system a) air/(d-cer/h-chol/h-BA) monolayer/NRW and b) air/(d-cer/h-chol/d-BA) monoalyer/NRW without and with the presence of nanogels $30 \mathrm{MBA}$ at $37^{\circ} \mathrm{C}$. Both mixed lipid monolayers are spread at $31.0 \mathrm{mN} \mathrm{m}^{-1}$. The solid lines are fits to the data.

Table 4 Parameters used for fits to system air/(d-cer/h-chol/d-BA)/NRW without and with the presence of nanogels 30 MBA. The calculated fits are shown by solid lines in Fig. 5b.

\begin{tabular}{lccccc}
\hline Sample & $\begin{array}{c}\text { Temp, } \\
\pm 1{ }^{\circ} \mathrm{C}\end{array}$ & $\begin{array}{c}d, \AA \\
\pm 1\end{array}$ & $\begin{array}{c}N b_{\text {fitted }} \times 10^{-6} \AA^{-2} \\
\pm 0.1\end{array}$ & $\begin{array}{c}\sigma, \AA \\
\pm 1\end{array}$ & $\begin{array}{c}\text { Air-film } \\
\text { roughness, } \\
\AA \\
\pm 1\end{array}$ \\
\hline d-cer/h-chol/d-BA monolayer & 37 & 28 & 2.2 & 2 & 2 \\
d-cer/h-chol/d-BA monolayer + 30 MBA & 37 & 28 & 2.0 & 2 & 2 \\
\hline
\end{tabular}

$d$ - layer thickness, $N b_{f i t t e d}$ - fitted scattering length density, $\sigma$ - roughness 


\section{Interaction of nanogels with behenic acid monolayer}

The above results suggest that behenic acid is the main component in the mixed lipid monolayer to interact with nanogels at $31.0 \mathrm{mN} \mathrm{m}^{-1}$ and $37{ }^{\circ} \mathrm{C}$, indicating the important role that fatty acid may play for the transport across biological membranes. Therefore, the next step focused on the study of the interaction of nanogels with a behenic acid monolayer.

The thermo-stability of bare d-behenic acid (d-BA) monolayer (molecular area of $40 \AA^{2}$ ) over the temperature range of $25-45^{\circ} \mathrm{C}$ was first examined by NR and the results (Fig. S9) suggest that the behenic acid monolayer is stable over the investigated temperature range. The reflectivity data can be fitted to a one-layer model. The fitted thickness of the monolayer is equal to $25 \AA$, which is shorter than the fully extended molecule length (28 $\AA$ ), suggesting that the molecules are tilted at the air-water interface. The tilted angle relative to the normal of the interface was calculated to be $27^{\circ}$, which is consistent with published literature. ${ }^{51}$ The volume fraction of dbehenic acid $\varphi_{d-B A}$ was calculated to be $78 \%$ (Equation 2), indicating a relatively coarse packing of the molecules.

The interaction of nanogels $30 \mathrm{MBA}$ with d-behenic acid monolayer at air-NRW interface was then investigated as a function of temperature from 25 to $45{ }^{\circ} \mathrm{C}$. The normalised neutron reflectivity profiles as a function of scattering wave vector $Q\left(\AA^{-1}\right)$ before and after the addition of $30 \mathrm{MBA}$ are shown in Fig. 6a. It was found that all the reflectivity datasets can be represented by a one-layer model. The fits are shown by solid lines in Fig. 6a and the corresponding parameters are given in Table 5. The $N b$ profiles are shown in Fig. $6 \mathrm{~b}$.

The scattering length density $N b$ dramatically decreases from $4.4 \times 10^{-6} \AA^{-2}$ to $0.4 \times 10^{-6} \AA^{-2}$ (Table 5) upon addition of $30 \mathrm{MBA}$, suggesting a loss of a large amount of deuterated behenic acids from the interface as a result of their association with nanogels. As the behenic acids were 
replaced by the newly formed behenic-nanogel complexes at the interface (schematically shown in Fig. 6c-f), the layer thickness increases from $25 \AA$ to $43 \AA$ upon the addition of the nanogels. The increase in the NR incoherent background (Fig. 6a) also suggests the presence of increased amount of protonated materials (i.e. nanogels and behenic-nanogel complexes) at the air-NRW interface.

The $N b$ of the layer increases with temperature up to $37{ }^{\circ} \mathrm{C}\left(N b=0.7 \times 10^{-6} \AA^{-2}\right)$ and it then decreases to $0.6 \times 10^{-6} \AA^{-2}$ as temperature further increases to $45^{\circ} \mathrm{C}$. This decrease in $\mathrm{Nb}$ at $45^{\circ} \mathrm{C}$ suggests loss of materials at the interface and is attributed to a possible aggregation and the subsequent precipitation (observed at the bottom of the Langmuir trough after the experiment) taking place at the elevated $45^{\circ} \mathrm{C}$ (Fig. 6f). This phenomenon is different from the adsorption of pure $30 \mathrm{MBA}$ at the air-water interface, which showed increased amount of adsorbed nanogels at the interface above $44^{\circ} \mathrm{C}$ with no aggregation (Fig. 1).

The above results suggest a stronger ability of nanogels to interact with the behenic acid monolayer than with the ceramide lipid monolayer. This may be tentatively associated with the behenic acid having one hydrocarbon chain and being more flexible hence it can easily be accommodated within the hydrophobic domains of the nanogels. As a consequence, it is easier for nanogels to disrupt the packing of the behenic acids in the monolayer and deplete the molecules from the interface. 

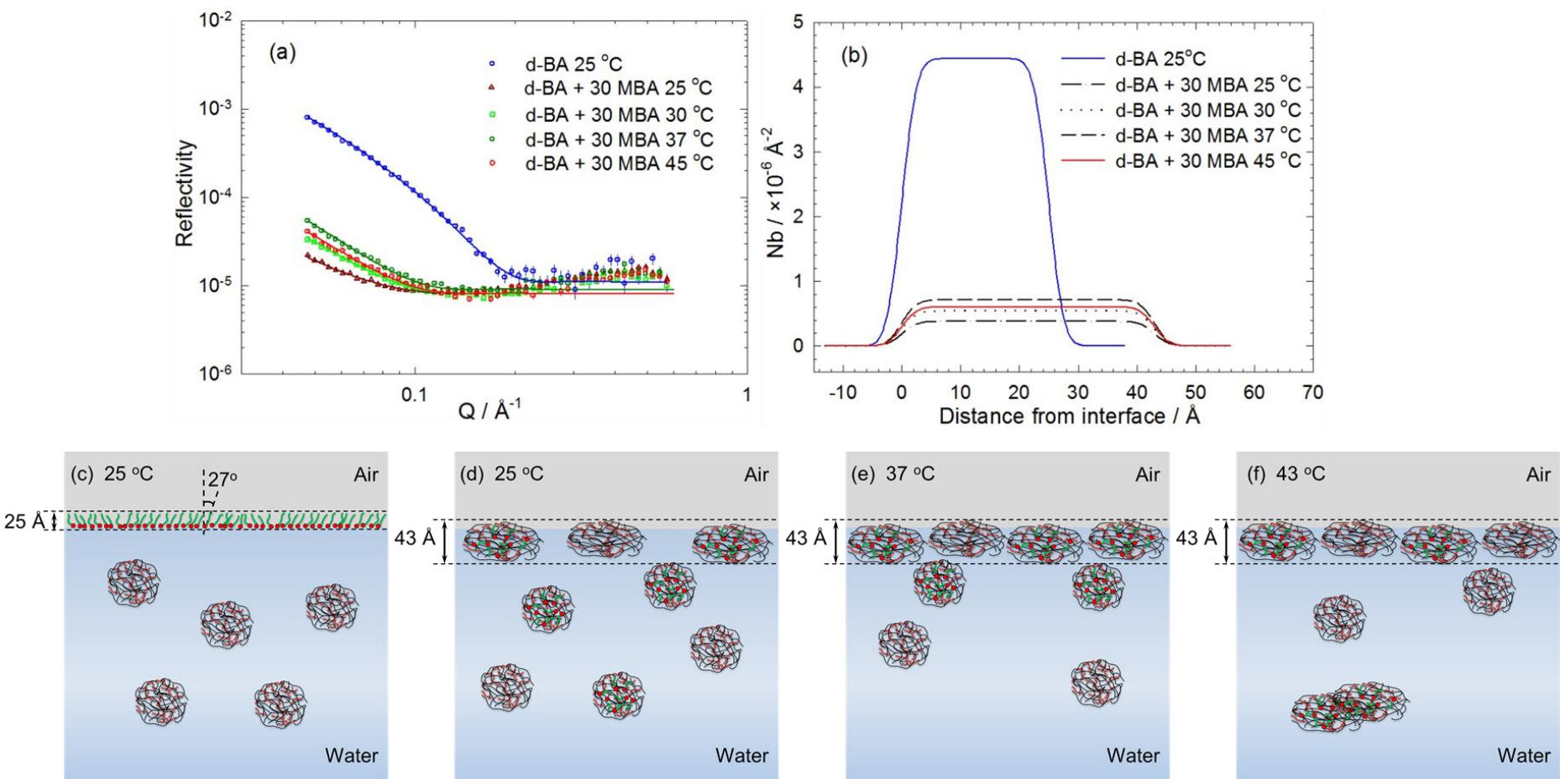

Fig. 6 a) Reflectivity spectra $R(Q)$ from the system air/d-BA monolayer/NRW for a series of temperatures without and with the presence of $30 \mathrm{MBA}$. The solid lines are fits to the data using one-layer model; b) Scattering length density $(\mathrm{Nb})$ profiles for calculated reflectivity profiles shown in a). c) - f) Schematic diagrams show the interaction between nanogels $30 \mathrm{MBA}$ and the behenic acid monolayer (molecular area of $40 \AA^{2}$ ) at the air-water interface. After adding 30 MBA, the behenic acids are depleted by the nanogels to form water dispersible complexes. The adsorption of these complexes and nanogels at the interface at $25{ }^{\circ} \mathrm{C}, 37{ }^{\circ} \mathrm{C}$ and $45{ }^{\circ} \mathrm{C}$ is shown in d) - f), respectively. 
Table 5 Parameters used for fits to system air/d-BA/NRW without and with the presence of nanogels 30 MBA. The calculated fits are shown by solid lines in Fig. 6a.

\begin{tabular}{lccccc}
\hline Sample & $\begin{array}{c}\text { Temp, } \\
\pm 1{ }^{\circ} \mathrm{C}\end{array}$ & $\begin{array}{c}d, \AA \\
\pm 1\end{array}$ & $\begin{array}{c}N b_{\text {fitted }} \times 10^{-6} \AA^{-2} \\
\pm 0.1\end{array}$ & $\begin{array}{c}\sigma, \AA \\
\pm 1\end{array}$ & $\begin{array}{c}\text { Air-film } \\
\text { roughness, } \AA \\
\pm 1\end{array}$ \\
\hline d-BA monolayer & 25 & 25 & 4.44 & 2 & 2 \\
d-BA monolayer + 30 MBA & 25 & 43 & 0.38 & 2 & 2 \\
d-BA monolayer + 30 MBA & 30 & 43 & 0.55 & 2 & 2 \\
d-BA monolayer + 30 MBA & 37 & 43 & 0.71 & 2 & 2 \\
d-BA monolayer + 30 MBA & 45 & 43 & 0.60 & 2 & 2 \\
\hline
\end{tabular}

$d$ - layer thickness, $N b_{\text {fitted }}$ - fitted scattering length density, $\sigma-$ roughness

\section{Conclusion}

We have shown in this work that the adsorption of NIPAM nanogels (30 MBA) at the air-water interface is temperature dependent and reversible. It was found that the interaction of these nanogels with lipid monolayers at the air-water interface is largely dependent on the monolayer fluidity. A pure ceramide lipid monolayer compressed to $42 \AA^{2}\left(31.0 \mathrm{mN} \mathrm{m}^{-1}\right)$ showed no evidence of any substantial interaction with the nanogels. This is due to the formation of a highly ordered crystalline phase with lipid molecules oriented perpendicular to the air-water interface at $42 \AA^{2}$, leading to poor monolayer fluidity. After the molecule area increased to $44 \AA^{2}(20.0 \mathrm{mN}$ $\mathrm{m}^{-1}$ ), a loosely packed phase with larger monolayer fluidity emerged. The introduction of nanogels underneath this monolayer led to the depletion of lipids from the interface as a result of the formation of water dispersible ceramide-nanogel complexes. This complex formation was further facilitated with increase of temperature and was found to be irreversible, indicative of a strong bonding between nanogels and ceramide lipids. 
However, in the case of ceramide/cholesterol/behenic acid mixed lipid monolayer at surface pressure of $31.0 \mathrm{mN} \mathrm{m}^{-1}$, it was found that nanogels were able to interact strongly with the mixed monolayer. This is because of the increase in the fluidity of the monolayer upon introduction of cholestrol and behenic acid ${ }^{52}$. This increase in monolayer fluidity encourages the interaction of nanogels with monolayer components. Behenic acids in particular was found to be the component displaying the strongest interaction with nanogels. This finding was further demonstrated by the experiment involving nanogels with a pure behenic acid monolayer. The results indicate the important role of fatty acids in facilitating membrane transport. These data presented therefore suggest that the formation of complexes, as a result of the interaction between nanogels and different components of a membrane, represents a step in the possible mechanism by which they can permeate through biological barriers.

\section{Supporting Information}

Nanogel synthesis and characterisation; molecular structure and scattering length density of lipids used; surface pressure molecular area $(\Pi-A)$ isotherms of ceramide lipid and behenic acid at $25{ }^{\circ} \mathrm{C}$; temperature dependence of meso-equilibrium surface pressure of $30 \mathrm{MBA}$ aqueous dispersion; BAM images of $30 \mathrm{MBA}$ aqueous dispersion, ceramide lipid monolayer with and without the presence of $30 \mathrm{MBA}$ at the air-water interface at different temperatures; NR measurements of bare ceramide lipid monolayer and behenic acid monolayer as a function of temperature at the air-water interface (PDF) 


\section{AUTHOR INFORMATION}

\section{Corresponding Author}

*E-mail a.zarbakhsh@qmul.ac.uk; Tel +44 (0)20 78823259.

*E-mail m.resmini@gmul.ac.uk; Tel +44 (0)20 78823268.

Notes

The authors declare no competing financial interest.

\section{ACKNOWLEDGEMENT}

The authors wish to thank the STFC, UK, for beam time on SURF (ISIS) and the European Commission (FP7 Marie Curie Actions, projects NANODRUG, MCITN-2011-289554) for financial support. Queen Mary, University of London and Chinese Scholarship Council are gratefully acknowledged for funding a PhD studentship to HS.

\section{REFERENCES}

(1) Wertz, P. W. Lipids and barrier function of the skin. Acta Derm.Venereol.-Suppl. 2000, (208), 7-11.

(2) Baroli, B. Penetration of nanoparticles and nanomaterials in the skin: fiction or reality? $J$. Pharm. Sci. 2010, 99 (1), 21-50.

(3) Barry, B. W. Novel mechanisms and devices to enable successful transdermal drug delivery. Eur. J. Pharm. Sci. 2001, 14 (2), 101-114.

(4) Talreja, P. S.; Kasting, G. B.; Kleene, N. K.; Pickens, W. L.; Wang, T.-F. Visualization of the lipid barrier and measurement of lipid pathlength in human stratum corneum. AAPS Pharmsci 2001, 3 (2), 48-56. 
(5) Trommer, H.; Neubert, R. Overcoming the stratum corneum: the modulation of skin penetration. Skin Pharmacol. Physiol. 2006, 19 (2), 106-121.

(6) Bouwstra, J. A.; Gooris, G. S.; Cheng, K.; Weerheim, A.; Bras, W.; Ponec, M. Phase behavior of isolated skin lipids. J. Lipid Res. 1996, 37 (5), 999-1011.

(7) Jung, T.; Kamm, W.; Breitenbach, A.; Kaiserling, E.; Xiao, J.; Kissel, T. Biodegradable nanoparticles for oral delivery of peptides: is there a role for polymers to affect mucosal uptake? Eur. J. Pharm. Biopharm. 2000, 50 (1), 147-160.

(8) Langer, R. Drugs on target. Science 2001, 293 (5527), 58-59.

(9) Okeyoshi, K.; Suzuki, D.; Kishimura, A.; Yoshida, R. Photoinduced Hydrogen Generating Nanogel Systems. Small 2011, 7 (3), 311-315.

(10) Peppas, N. A.; Hilt, J. Z.; Khademhosseini, A.; Langer, R. Hydrogels in biology and medicine: from molecular principles to bionanotechnology. Adv. Mater. 2006, 18 (11), 13451360.

(11) Bysell, H.; Månsson, R.; Hansson, P.; Malmsten, M. Microgels and microcapsules in peptide and protein drug delivery. Adv. Drug Deliv. Rev. 2011, 63 (13), 1172-1185.

(12) Malmsten, M.; Bysell, H.; Hansson, P. Biomacromolecules in microgels-Opportunities and challenges for drug delivery. Curr. Opin. Coll. Interface Sci. 2010, 15 (6), 435-444.

(13) Oh, J. K.; Drumright, R.; Siegwart, D. J.; Matyjaszewski, K. The development of microgels/nanogels for drug delivery applications. Prog. Polym. Sci. 2008, 33 (4), 448-477. 
(14) Sahiner, N.; Godbey, W.; McPherson, G. L.; John, V. T. Microgel, nanogel and hydrogelhydrogel semi-IPN composites for biomedical applications: synthesis and characterization. Colloid. Polym. Sci. 2006, 284 (10), 1121-1129.

(15) Rejinold, N. S.; Baby, T.; Chennazhi, K.; Jayakumar, R. Dual drug encapsulated thermosensitive fibrinogen-graft-poly ( $\mathrm{N}$-isopropyl acrylamide) nanogels for breast cancer therapy. Colloids Surf. B: Biointerfaces 2014, 114, 209-217.

(16) Zha, L.; Banik, B.; Alexis, F. Stimulus responsive nanogels for drug delivery. Soft Matter 2011, 7 (13), 5908-5916.

(17) Das, D.; Ghosh, P.; Ghosh, A.; Haldar, C.; Dhara, S.; Panda, A. B.; Pal, S. StimulusResponsive, Biodegradable, Biocompatible, Covalently Cross-Linked Hydrogel Based on Dextrin and Poly (N-isopropylacrylamide) for in Vitro/in Vivo Controlled Drug Release. $A C S$ Appl. Mater. Interfaces 2015, 7 (26), 14338-14351.

(18) Saikia, A.; Aggarwal, S.; Mandal, U. Preparation and Controlled Drug Release Characteristics of Thermoresponsive PEG/Poly (NIPAM-co-AMPS) Hydrogels. Int. J. Polymer. Mater. 2013, 62 (1), 39-44.

(19) Schmaljohann, D. Thermo-and pH-responsive polymers in drug delivery. Adv. Drug Deliv. Rev. 2006, 58 (15), 1655-1670.

(20) Gruner, S. M. Intrinsic curvature hypothesis for biomembrane lipid composition: a role for nonbilayer lipids. Proc. Natl. Acad. Sci. 1985, 82 (11), 3665-3669.

(21) Domenici, F.; Castellano, C.; Dell'Unto, F.; Albinati, A.; Congiu, A. Colloids and Surfaces B: Biointerfaces. 2011, 88, 432-439 
(22) Darre, L.; Iglesias-Fernandez, J.; Kohlmeyer, A.; Wacklin, H.; Domene, C. J. Chem. Theory Comput, 2005, 11 4875-4884.

(23) Brezesinski, G.; Möhwald, H. Langmuir monolayers to study interactions at model membrane surfaces. Adv. Colloid Interface Sci. 2003, 100, 563-584.

(24) Sun, S.; Hu, J.; Tang, H.; Wu, P. Spectral interpretation of thermally irreversible recovery of poly (N-isopropylacrylamide-co-acrylic acid) hydrogel. Phys. Chem. Chem. Phys. 2011, 13 (11), 5061-5067.

(25) Lopez, V. C.; Hadgraft, J.; Snowden, M. The use of colloidal microgels as a (trans) dermal drug delivery system. Int. J. Pharm. 2005, 292 (1), 137-147.

(26) Lopez, V. C.; Raghavan, S.; Snowden, M. Colloidal microgels as transdermal delivery systems. React. Funct. Polym. 2004, 58 (3), 175-185.

(27) Samah, N. A.; Williams, N.; Heard, C. M. Nanogel particulates located within diffusion cell receptor phases following topical application demonstrates uptake into and migration across skin. Int. J. Pharm. 2010, 401 (1), 72-78.

(28) Samah, N. H. A.; Heard, C. M. Enhanced in vitro transdermal delivery of caffeine using a temperature-and pH-sensitive nanogel, poly (NIPAM-co-AAc). Int. J. Pharm. 2013, 453 (2), 630-640.

(29) Singka, G. S. L.; Samah, N. A.; Zulfakar, M. H.; Yurdasiper, A.; Heard, C. M. Enhanced topical delivery and anti-inflammatory activity of methotrexate from an activated nanogel. Eur. J. Pharm. Biopharm. 2010, 76 (2), 275-281. 
(30) Rancan, F.; Blume-Peytavi, U.; Vogt, A. Utilization of biodegradable polymeric materials as delivery agents in dermatology. Clin. Cosmet. Investig. Dermatol. 2014, 7, 23-34.

(31) Zielińska, K.; Sun, H.; Campbell, R. A.; Zarbakhsh, A.; Resmini, M. Smart nanogels at the air/water interface: structural studies by neutron reflectivity. Nanoscale 2016, 8, 4951-4960.

(32) Penfold, J. Neutron reflectivity and soft condensed matter. Curr. Opin. Coll. Interface Sci. 2002, 7 (1), 139-147.

(33) Wacklin, H. P. Neutron reflection from supported lipid membranes. Curr. Opin. Coll. Interface Sci. 2010, 15 (6), 445-454.

(34) C. M. Hollinshead; R. D. Harvey; D. J. Barlow; J. R. P. Webster; A. V. Hughes; A. Weston; Lawrence, M. J. Effects of Surface Pressure on the Structure of Distearoylphosphatidylcholine Monolayers Formed at the Air/Water Interface. Langmuir 2009, 25 (25), 4070-4077.

(35) Krueger, S. Neutron reflection from interfaces with biological and biomimetic materials. Curr. Opin. Coll. Interface Sci. 2001, 6 (6), 111-117.

(36) Penfold, J.; Richardson, R.; Zarbakhsh, A.; Webster, J.; Bucknall, D.; Rennie, A.; Jones, R.; Cosgrove, T.; Thomas, R.; Higgins, J. Recent advances in the study of chemical surfaces and interfaces by specular neutron reflection. J. Chem. Soc., Faraday Trans. 1997, 93 (22), 38993917.

(37) Campana, M.; Webster, J. R.; Lawrence, M. J.; Zarbakhsh, A. Structural conformation of lipids at the oil-water interface. Soft Matter 2012, 8 (34), 8904-8910.

(38) Heavens, O. S. Optical properties of thin solid films. Courier Corporation: 1991. 
(39) Brugger, B.; Rütten, S.; Phan, K. H.; Möller, M.; Richtering, W. The colloidal suprastructure of smart microgels at oil-water interfaces. Angew. Chem. Int. Ed. 2009, 48 (22), 3978-3981.

(40) Destribats, M.; Lapeyre, V.; Wolfs, M.; Sellier, E.; Leal-Calderon, F.; Ravaine, V.; Schmitt, V. Soft microgels as Pickering emulsion stabilisers: role of particle deformability. Soft Matter 2011, 7 (17), 7689-7698.

(41) Geisel, K.; Isa, L.; Richtering, W. Unraveling the 3D localization and deformation of responsive microgels at oil/water interfaces: a step forward in understanding soft emulsion stabilizers. Langmuir 2012, 28 (45), 15770-15776.

(42) Richtering, W. Responsive emulsions stabilized by stimuli-sensitive microgels: emulsions with special non-Pickering properties. Langmuir 2012, 28 (50), 17218-17229.

(43) Lee, L.; Jean, B.; Menelle, A. Effect of temperature on the adsorption of poly (nisopropylacrylamide) at the air-solution interface. Langmuir 1999, 15 (9), 3267-3272.

(44) Monteux, C.; Marliere, C.; Paris, P.; Pantoustier, N.; Sanson, N.; Perrin, P. Poly (Nisopropylacrylamide) Microgels at the Oil- Water Interface: Interfacial Properties as a Function of Temperature. Langmuir 2010, 26 (17), 13839-13846.

(45) Richardson, R. M.; Pelton, R.; Cosgrove, T.; Zhang, J. A neutron reflectivity study of poly (N-isopropylacrylamide) at the air-water interface with and without sodium dodecyl sulfate. Macromolecules 2000, 33 (17), 6269-6274.

(46) Löfgren, H.; Pascher, I. Molecular arrangements of sphingolipids. The monolayer behaviour of ceramides. Chem. Phys. Lipids 1977, 20 (4), 273-284. 
(47) Fanani, M. L.; Maggio, B. Phase state and surface topography of palmitoyl-ceramide monolayers. Chem. Phys. Lipids 2010, 163 (6), 594-600.

(48) Shah, J.; Atienza, J. M.; Duclos, R.; Rawlings, A. V.; Dong, Z.; Shipley, G. Structural and thermotropic properties of synthetic C16: 0 (palmitoyl) ceramide: effect of hydration. J. Lipid Res. 1995, 36 (9), 1936-1944.

(49) Scheffer, L.; Solomonov, I.; Weygand, M. J.; Kjaer, K.; Leiserowitz, L.; Addadi, L. Structure of cholesterol/ceramide monolayer mixtures: implications to the molecular organization of lipid rafts. Biophys. J. 2005, 88 (5), 3381-3391.

(50) Lande, M. B.; Donovan, J. M.; Zeidel, M. L. The relationship between membrane fluidity and permeabilities to water, solutes, ammonia, and protons. J. Gen. Physiol. 1995, 106 (1), 6784.

(51) Bommarito, G.; Foster, W.; Pershan, P. S.; Schlossman, M. A determination of the phase diagram of relaxed Langmuir monolayers of Behenic acid. J. Chem. Phys. 1996, 105 (12), 52655284.

(52) Kenn, R. M.; Boehm, C.; Bibo, A. M.; Peterson, I. R.; Moehwald, H.; Als-Nielsen, J.; Kjaer, K. Mesophases and crystalline phases in fatty acid monolayers. J. Phys. Chem 1991, 95 (5), 2092-2097. 